\title{
A quantitative feeding assay in adult Drosophila reveals rapid modulation of food ingestion by its nutritional value
}

\author{
Wei $\mathrm{Qi}^{1,2+}$, Zhe Yang ${ }^{1,2+}$, Ziao Lin ${ }^{1,2}$, Jin-Yong Park ${ }^{3}$, Greg S. B. Suh ${ }^{3}$ and Liming Wang ${ }^{1,2^{*}}$
}

\begin{abstract}
Background: Food intake of the adult fruit fly Drosophila melanogaster, an intermittent feeder, is attributed to several behavioral elements including foraging, feeding initiation and termination, and food ingestion. Despite the development of various feeding assays in fruit flies, how each of these behavioral elements, particularly food ingestion, is regulated remains largely uncharacterized.

Results: To this end, we have developed a manual feeding (MAFE) assay that specifically measures food ingestion of an individual fly completely independent of the other behavioral elements. This assay reliably recapitulates the effects of known feeding modulators, and offers temporal resolution in the scale of seconds. Using this assay, we find that fruit flies can rapidly assess the nutritional value of sugars within 20-30 s, and increase the ingestion of nutritive sugars after prolonged periods of starvation. Two candidate nutrient sensors, SLC5A11 and Gr43a, are required for discriminating the nutritive sugars, D-glucose and D-fructose, from their non-nutritive enantiomers, respectively. This suggests that differential sensing mechanisms play a key role in determining food nutritional value.
\end{abstract}

Conclusions: Taken together, our MAFE assay offers a platform to specifically examine the regulation of food ingestion with excellent temporal resolution, and identifies a fast-acting neural mechanism that assesses food nutritional value and modulates food intake.

Keywords: Starvation, Nutritive sugar, Feeding, Drosophila

\section{Background}

Energy homeostasis of all animal species relies on a precise balance between energy intake and expenditure. For intermittent feeders including humans, feeding behavior usually begins with off-food foraging, followed by one or multiple rounds of feeding initiation, food ingestion, and feeding termination before leaving food source and starting a new round of foraging (Fig. 1a) [1]. Although the neural control of food intake has been extensively studied in rodent and insect model organisms [2], it is often unclear whether the modulation of food intake occurs

\footnotetext{
* Correspondence: Imwang83@zju.edu.cn

${ }^{\dagger}$ Equal contributors

'Life Sciences Institute, Zhejiang University, Hangzhou, Zhejiang 310058, China

${ }^{2}$ Innovation Center for Cell Signaling Network, Zhejiang University, Hangzhou, Zhejiang 310058, China

Full list of author information is available at the end of the article
}

through changes in foraging, feeding initiation and termination, food ingestion, or combinations of these elements. To better understand the neural regulation of energy homeostasis, it is critical to investigate how these behavioral elements of food intake are independently regulated.

A wealth of behavioral assays have been developed to quantify different aspects of feeding behavior in the adult fruit fly Drosophila melanogaster, which is also an intermittent feeder [3]. Starvation-induced hyperactivity and odor-driven food search have been used as measurements of foraging activity in flies $[4,5]$. Two recently developed automated assays, named FLIC (Fly Liquid-Food Interaction Counter) and flyPAD (fly Proboscis and Activity Detector), quantify the physical contact of flies' proboscis and food surface through monitoring the changes in conductance and capacitance, respectively [6,7]. These assays therefore offer 


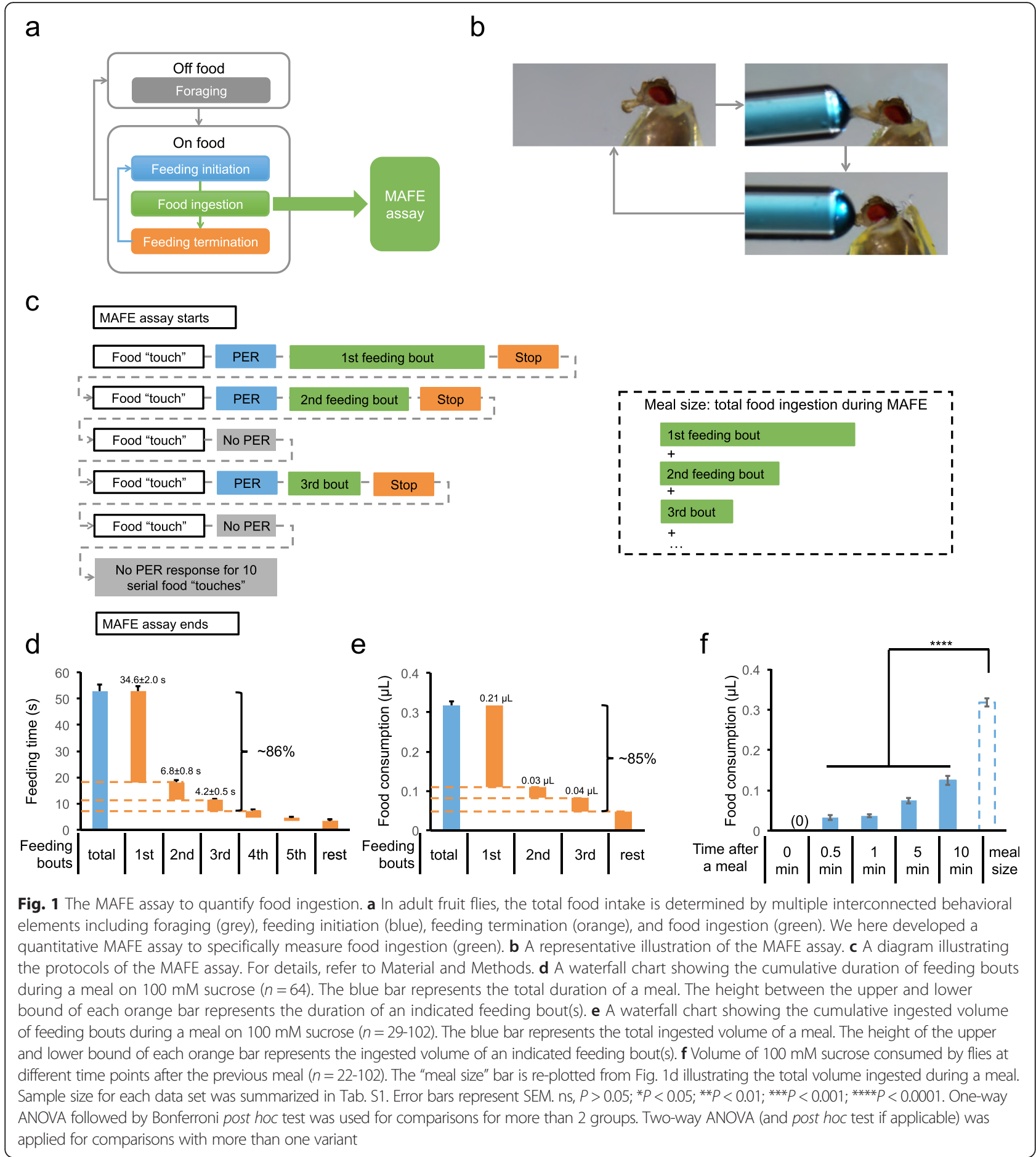

quantitative measurements of feeding initiation, such as the frequency of the proboscis extension reflex (PER) responses. However, a behavioral assay that specifically measures food ingestion without influence of the other behavioral elements of food intake still lacks. Labeling food with radioactive or colored substrates has been used to measure food ingestion of a group of flies [8, 9]. These methods, however, only offer semi-quantitative measurements of food ingestion and preference, and lack the resolution at individual flies. The capillary feeder (CAFE) assay continuously and reliably detects the volume of liquid food being ingested [10]. But foraging behavior becomes a major confound because the CAFE setup requires flies to 
reliably locate food source at a tiny tip of a hanging capillary [3], a behavior task that is not natural to flies and requires foraging capability. This assay also requires a longer time course (i.e. hours to days) [3].

We have herein developed a novel and quantitative feeding assay, named MAFE assay, to address the caveats associated with previous feeding assays. The MAFE assay quantifies food ingestion without the interference from foraging and feeding initiation, and offers temporal resolution in the scale of seconds. We find that the MAFE assay reliably recapitulates the effects of known feeding modulators including food deprivation and gut stretch. Using this assay, we further uncover that flies can detect the nutritional value of $\mathrm{D}$-glucose and $\mathrm{D}$ fructose, the two dietary sugars for fruit flies, and increase food ingestion after prolonged periods of starvation $[11,12]$. Intriguingly, the discrimination of these nutritive sugars from their non-nutritive enantiomers only takes $20-30$ s, and requires two candidate nutrient sensors, SLC5A11 and Gr43a, respectively $[12,13]$. This suggests that a fast-acting mechanism plays a critical role in determining the nutritional value of sugars in Drosophila.

\section{Results and discussion}

\section{The development of the MAFE assay}

We developed a quantitative MAFE assay to specifically measure food ingestion in an adult fly (Fig. 1a-c). An individual fly was immobilized in a $200 \mu \mathrm{L}$ pipette tip (Fig. 1b, left), and presented with liquid food filled in a graduated glass capillary. In successful feeding events, flies started feeding by extending their proboscis into the food (Fig. 1b, middle). After we confirmed that flies had normal PER responses to food, we fed the flies until they stopped responding to a series of ten food stimuli (Fig. 1b, right). The total volume of liquid food consumed between each round of feeding initiation and termination would be a measurement of food ingestion (Fig. 1c). Hence, the MAFE assay specifically measures food ingestion independently from potential changes in foraging behavior. As PER and food consumption can be separately assayed in the MAFE assay, it can also separate the effects on food ingestion from those on feeding initiation (for example, see Fig. 3a-b).

Having established this assay, we next determined the kinetics of food ingestion revealed by the MAFE assay. Flies starved for $36 \mathrm{~h}$ usually exhibited several bouts of feeding behavior before they stopped responding to food stimuli. The duration and ingested volume of each feeding bout was recorded. As shown in Fig. 1d, 36-h starved flies spent $52.8 \pm 2.6 \mathrm{~s}$ on average in feeding when presented with sucrose. The first feeding bout took $34.6 \pm$ $2.0 \mathrm{~s}$, and the first three feeding bouts together made up to $\sim 86 \%$ of the total feeding duration (Fig. 1d). The starved flies ingested $0.32 \pm 0.01 \mu \mathrm{L}$ liquid food on average, and the first three feeding bouts together comprised up to $\sim 85 \%$ of the total feeding volume (Fig. 1e). These flies ingested significantly smaller volumes of food when presented with sucrose again in the next $10 \mathrm{~min}$ after the MAFE assay (Fig. 1f), suggesting that they remained satiated and uninterested in food for the following short period. These results indicate that the total volume of food ingestion during the course of our MAFE assay likely defines the size of a "meal" (Fig. 1c, right), and therefore can be used as a measurement to investigate the regulation of food ingestion. It is also worth noting that the meal size of fed flies in the MAFE assay (Fig. 2a) is similar to that in the CAFE assay [10], suggesting that food ingestion of immobilized flies is comparable to that of freely moving flies.

\section{The MAFE assay recapitulates the effect of food deprivation and gut stretch on food ingestion}

We asked whether the MAFE assay could reliably recapitulate the effect on food ingestion by known modulators of feeding behavior. Food deprivation promotes feeding behavior (also see Additional file 1: Figure S1a-b) [6, 14]. Consistently, we found that the length of starvation significantly increased food ingestion, from $0.10 \pm$ $0.01 \mu \mathrm{L}$ in fed flies to $0.21 \pm 0.02 \mu \mathrm{L}$ in 24 -h starved flies and $0.27 \pm 0.02 \mu \mathrm{L}$ in 36 -h starved flies (Fig. 2a).

We further asked how starvation increased food consumption: whether it promoted feeding speed or feeding duration, or both. To this end, we assayed the duration and ingested volume from the first feeding bout of $12-\mathrm{h}$ and 36-h starved flies. As shown in Fig. 2b, the ingested volume for each fly showed a modest correlation with the duration of the feeding bout. We also found that the feeding speed was not significantly changed by a longer period of starvation (Fig. 2c). Taken together, these results suggest that starvation enhances food ingestion via increased duration of feeding rather than increased speed.

Gut stretch has also been shown to suppress the total food intake over a 24-h period by the activation of $\mathrm{HGN1}^{+}$mechanosensory neurons innervating the gut [15]. Consistent with this, acute silencing of $\mathrm{HGN}^{+}$ neurons by ectopically expressing a temperature sensitive form of dynamin protein (Shibire ${ }^{\text {ts1 }}$ ) increased food ingestion in fed flies at a restrictive temperature (Fig. 2d), while acute activation of these neurons by ectopically expressing a temperature sensitive TRP channel (TRPA1) reduced food ingestion in starved flies (Fig. 2e) $[16,17]$. In conclusion, our MAFE assay reliably quantifies food ingestion of individual flies without a potential confound from other behavioral elements that also influence feeding behavior. 

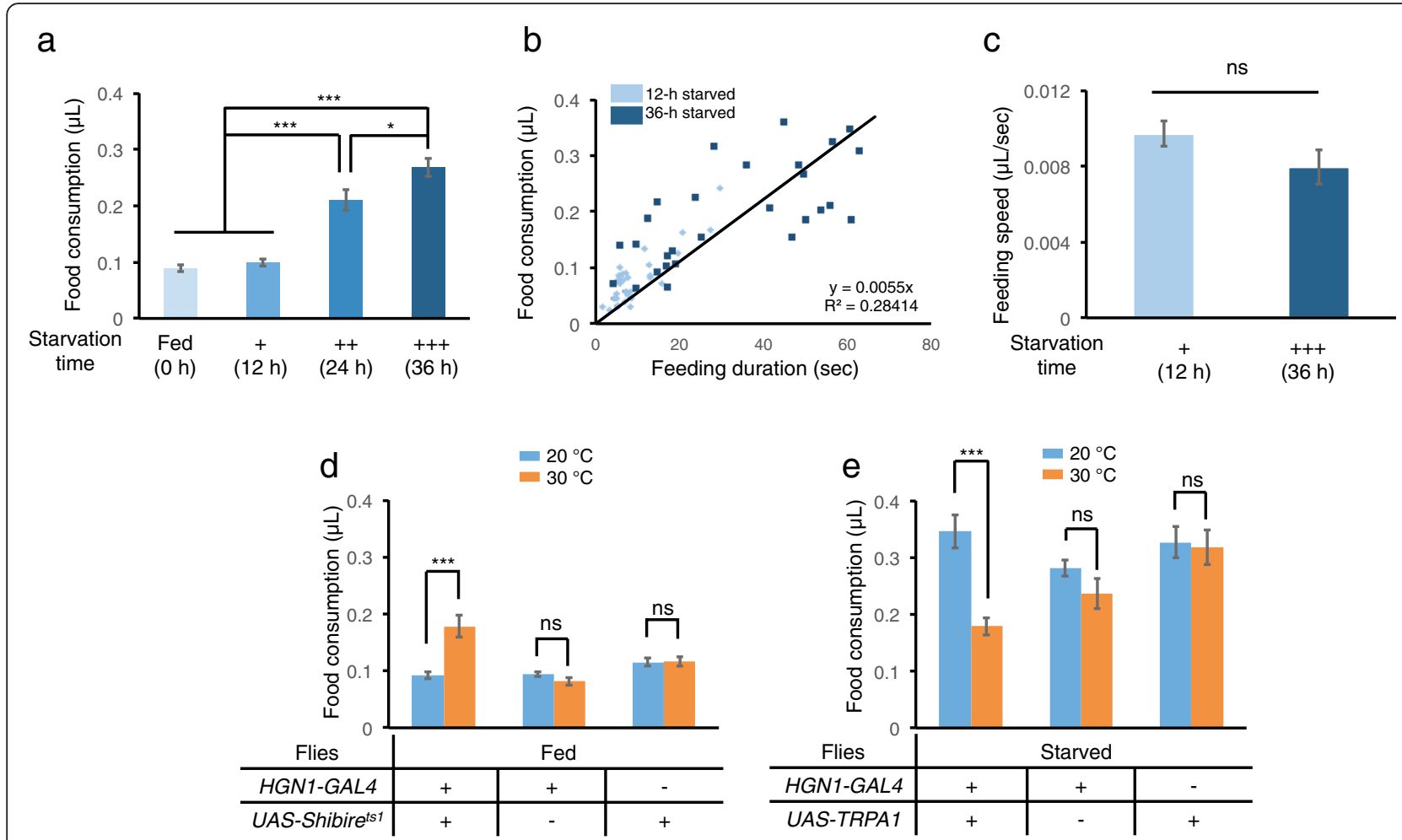

Fig. 2 The MAFE assay detects the effects of food deprivation and gut stretch on food ingestion. a Volume of $100 \mathrm{mM}$ sucrose consumed in a meal, by fed Canton-S flies, and those starved for 12, 24 and $36 \mathrm{~h}(n=25-29)$. b Duration ( $\mathrm{x}$-axis) and volume of $100 \mathrm{mM}$ sucrose consumed ( $\mathrm{y}$-axis) in the first feeding bout by Canton-S flies starved for 12 and $36 \mathrm{~h}(n=29-34)$. The regressed linear equation and the $\mathrm{R}^{2}$ value are listed in the plot. $\mathbf{c}$ Feeding speed of Canton-S flies starved for 12 and $36 \mathrm{~h}(n=29-34)$. $\mathbf{d}$ Volume of $100 \mathrm{mM}$ D-glucose consumed in a meal, by fed flies with indicated genotypes at $20^{\circ} \mathrm{C}$ or $30^{\circ} \mathrm{C}(n=24-39)$. e Volume of $100 \mathrm{mM}$ D-glucose consumed in a meal, by starved $(24-36 \mathrm{~h})$ flies with indicated genotypes at $20{ }^{\circ} \mathrm{C}$ or $30{ }^{\circ} \mathrm{C}(n=19-35)$. Sample size for each data set was summarized in Tab. S1. Error bars represent SEM. ns, $P>0.05$; ${ }^{*} P<0.05$; ${ }^{*} P<0.01$; ${ }^{* *} P<0.001$; ${ }^{* * *} P<0.0001$. One-way ANOVA followed by Bonferroni post hoc test was used for comparisons for more than 2 groups. Two-way ANOVA (and post hoc test if applicable) was applied for comparisons with more than one variant

\section{Rapid detection of nutritive sugars by flies after prolonged periods of starvation}

Recent reports have shown that fruit flies can detect the nutritional value of sugars and modulate food preference through a mechanism independent of peripheral gustatory perception (also see Additional file 1: Figure S1c-d) $[11,18]$. Because food preference was measured over the course of several hours in their assays, however, it was difficult to determine how fast flies can detect the caloric value of sugars and therefore, the underlying neurobiological and metabolic mechanisms. Because our MAFE assay offers a good temporal resolution (Fig. 1d), we sought to examine the effect of food nutritional value on food ingestion by using this assay.

D-glucose is one of the major carbohydrate sources for fruit flies [19]. L-glucose, its enantiomer, cannot be metabolized by flies and therefore, provides no nutritive value [20]. Nonetheless, both molecules evoked strong and comparable PER responses in starved flies, confirming that these two sugars are equally palatable to initiate feeding behavior (Fig. 3a) [11, 20]. We asked whether flies could respond differently to these two enantiomers during the course of a single meal. Using the MAFE assay, we found that 36-h starved flies ingested more than $50 \%$ volume on $\mathrm{D}$-glucose than on L-glucose $(0.27 \pm 0.02 \mu \mathrm{L}$ for $\mathrm{D}$-glucose and $0.17 \pm 0.01 \mu \mathrm{L}$ for $\mathrm{L}$-glucose), whereas flies starved for shorter time periods (12 and $24 \mathrm{~h}$ ) exhibited no difference (Fig. 3b). Similar effects were also observed for higher concentrations of D-/L-glucose (Additional file 1: Figure S2). Besides D-glucose, Dfructose is the other dietary sugar for flies [19]. Similar to D- and L-glucose, both D- and L-fructose enantiomers elicited comparable PER responses in starved flies (Fig. 3c). However, flies starved for 24 and $36 \mathrm{~h}$ consumed greater volumes of D-fructose than those of L-fructose (Fig. 3d).

Taken together, flies can detect the nutritional value of sugars, and enhance food ingestion of nutritive sugars upon food deprivation. Consistent with this hypothesis, increasing nutritional content of sugar enhanced food ingestion of 36-h starved flies in a dose-dependent manner (Fig. 3e). Most of the 36-h starved flies pre-fed with L-glucose still responded to subsequent re-stimulation 


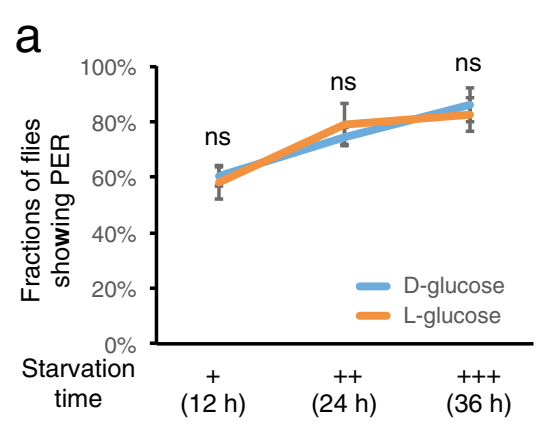

b

C

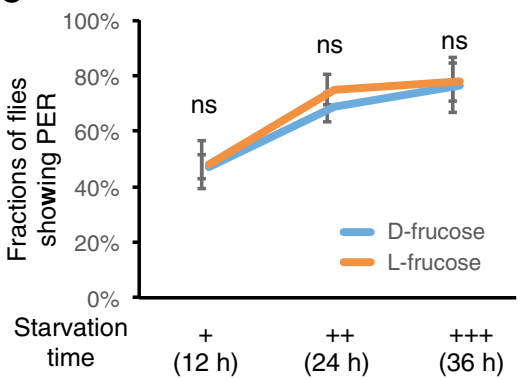

d

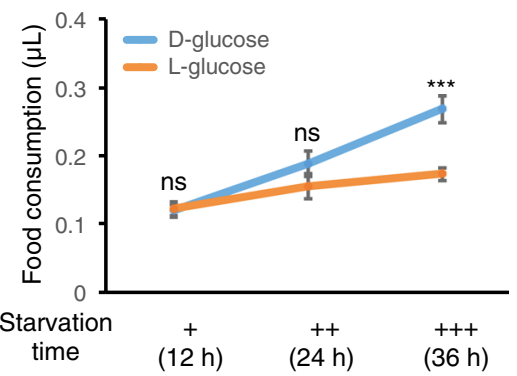

e
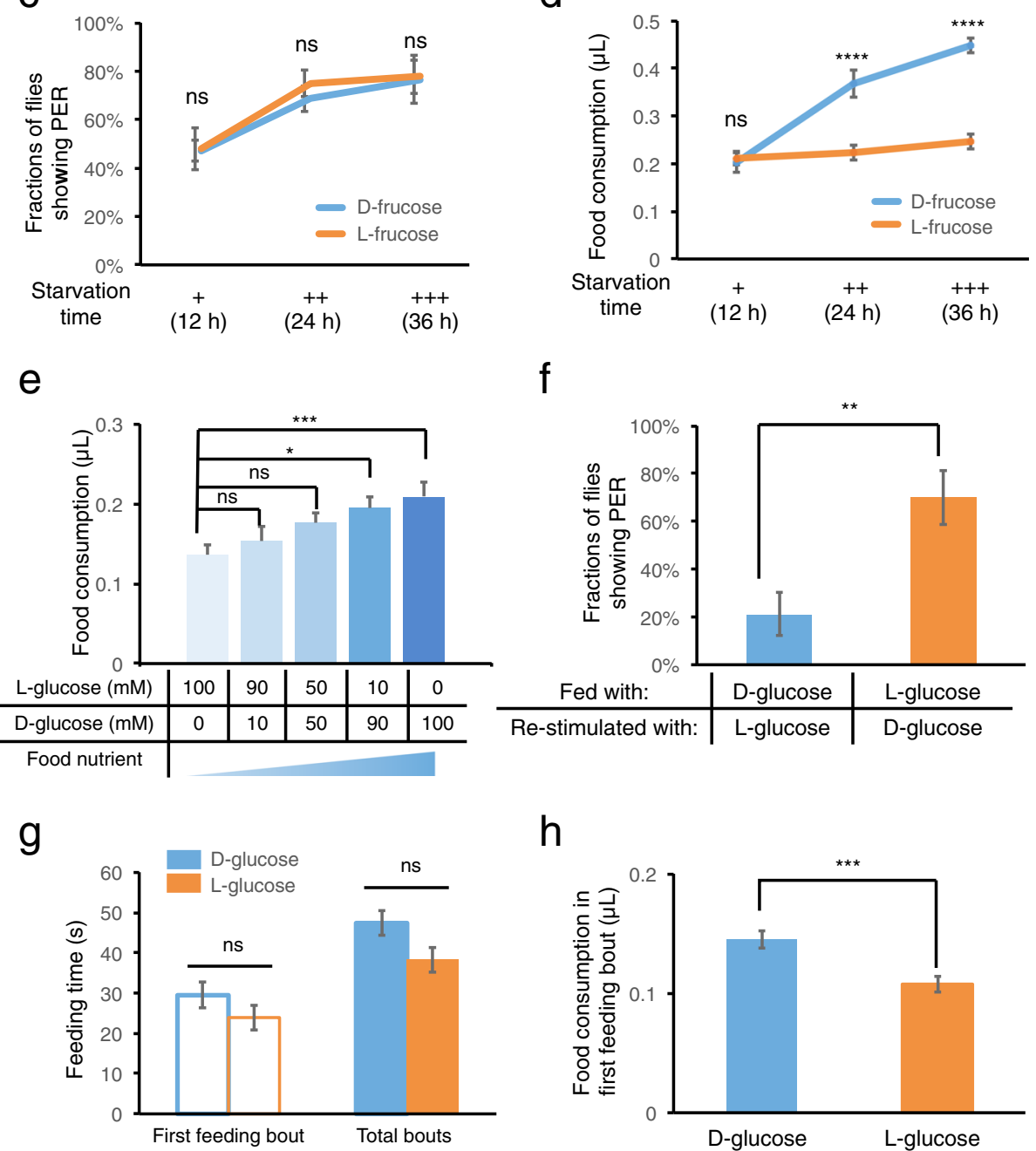

h

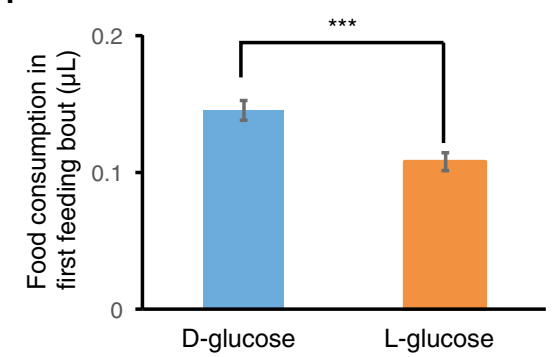

Fig. 3 Starved flies can assess food nutritional value and modulate food ingestion. a, c Fractions of Canton-S flies exhibiting PER responses to (a) $100 \mathrm{mM} \mathrm{D-/L-glucose}$ and (c) $100 \mathrm{mM}$ D-/L-fructose when starved for 12, 24, and $36 \mathrm{~h} \mathrm{(} n=19$-36). b, d Volume of (b) $100 \mathrm{mM} \mathrm{D-/L-glucose}$ and (d) $100 \mathrm{mM} \mathrm{D-/L-fructose} \mathrm{consumed} \mathrm{in} \mathrm{a} \mathrm{meal,} \mathrm{by} \mathrm{Canton-S} \mathrm{flies} \mathrm{starved} \mathrm{for} \mathrm{12,} 24$ and $36 \mathrm{~h}(n=19-36)$. e Volume of sugar solutions with indicated D- and L-glucose concentrations consumed in a meal, by 36-h starved Canton-S flies $(n=22-35)$. $\mathbf{f}$ Fractions of 36-h starved Canton-S flies exhibiting PER responses to the indicated second sugar ("Re-stimulated with") after finishing a meal with the indicated first sugar ("Fed with") $(n=25-26)$. $\mathbf{g}$ Feeding time of 36-h starved Canton-S flies when fed with $100 \mathrm{mM} \mathrm{D-/L-glucose}(n=24-30)$. $\mathbf{h}$ Volume of $100 \mathrm{mM} \mathrm{D-/L-glucose}$ consumed during the first feeding bout of a meal by 36-h starved Canton-S $(n=68-69)$. Sample size for each data set was summarized in Tab. S1. Error bars represent SEM. ns, $P>0.05$; ${ }^{*} P<0.05 ;{ }^{* *} P<0.01 ;{ }^{* *} P<0.001 ;{ }^{* * *} P<0.0001$. Student's $t$-test was used for pair wise comparisons. One-way ANOVA followed by Bonferroni post hoc test was used for comparisons for more than 2 groups. Two-way ANOVA (and post hoc test if applicable) was applied for comparisons with more than one variant 
of D-glucose, while starved flies pre-fed with D-glucose failed to respond to L-glucose, confirming that starved flies could be sated by nutritive D-glucose but not by non-nutritive L-glucose (Fig. 3f).

Intriguingly, starved flies could detect the nutritional value of sugars during the course of a single meal, which took $47.4 \pm 5.3 \mathrm{~s}$ on average for D-glucose and $38.2 \pm$ $3.1 \mathrm{~s}$ for L-glucose (Fig. 3g, "Total bouts"). We further found that 36-h starved flies consumed greater volumes of D-glucose than those of L-glucose during the first feeding bout of a meal (Fig. 3h, $0.14 \pm 0.01 \mu \mathrm{L}$ for Dglucose vs. $0.11 \pm 0.01 \mu \mathrm{L}$ for L-glucose); and it only took $29.5 \pm 3.3 \mathrm{~s}$ for D-glucose and $23.9 \pm 3.1 \mathrm{~s}$ for L-glucose (Fig. 3g, "First feeding bout"). These results highlight the presence of a fast-acting mechanism that detects food nutritional value and modulate food ingestion within 20-30 s. Our data are also consistent with the hypothesis that flies are capable of directly detecting nutritive sugar through a mechanism medicated by a small group of neuropeptidergic neurons in the fly brain [21].

\section{SLC5A11 and Gr43a mediates the discrimination of nutritive vs. non-nutritive sugars}

To further investigate the mechanism underlying the increased food ingestion of nutritive sugars, we examined two recently identified candidate food nutrient sensors, SLC5A11 and Gr43a, in our MAFE assay [12, 13]. SLC5A11, a brain-expressing $\mathrm{Na}^{+}$/solute co-transporter, is required for taste-independent nutrient selection in starved flies [13]. It was not clear, however, whether it was involved in the regulation of food ingestion per se. Unlike Canton-S controls (Fig. 4a), SLC5A11 ${ }^{1}$ hypomorphic mutant flies ingested comparable food volumes of D-glucose as those of L-glucose even after 36-h of starvation (Fig. 4b). This result indicates that SLC5A11 is required for discriminating nutritive $\mathrm{D}$-glucose from non-nutritive L-glucose after starvation.

Moreover, starved Canton-S flies, but not SLC5A11 ${ }^{1}$ mutants, exhibited a preference towards an odorant associated with nutritive D-glucose over another odorant associated with L-glucose in an appetitive olfactory conditioning assay. This suggests that starved flies are able to sense the difference between D-glucose and L-glucose in an SLC5A11 dependent manner (Additional file 1: Figure S3). Consistent with the observation using the MAFE assay, flies can quickly associate the nutritional content of sugar with an odorant within $2 \mathrm{~min}$. It remains unclear, however, whether SLC5A11 is involved in directly sensing the nutritional value of glucose, or regulating food ingestion. Gr43a, a brain-expressing gustatory receptor, was implied in the detection of hemolymph nutrient and the regulation of feeding behavior [12]. Gr43 ${ }^{\text {GALA }}$ mutant flies were still capable of discriminating D- vs. L-glucose when starved (Fig. 4c).
Interestingly, Gr43a, but not SLC5A11, was required for detecting the nutritional value associated with $\mathrm{D}$ fructose (Fig. 4d-f). These results highlight the difference in the mechanisms by which these molecules mediate behavior responses to different sugars (Fig. 4g).

In summary, we have developed a quantitative MAFE assay to investigate the regulation of food ingestion without the interference from other feeding related behavioral elements. Using this assay, we found that fruit flies could assess food nutritional value within $20-30 \mathrm{~s}$, and enhance food ingestion of nutritive sugars after prolonged starvation. Notably, the detection of D-glucose and D-fructose, two major dietary sugars for fruit flies in natural habitats, employs distinct neural mechanisms involving two candidate nutrient sensors SLC5A11 and Gr43a, respectively. Collectively, our study offers a starting point to further investigate how the food nutritional value influences different behavioral elements of feeding.

\section{Conclusions}

Here we describe a novel and quantitative feeding assay to examine the regulation of food ingestion precisely during the first bouts of feeding. Using the assay, we find that flies could detect the nutritional value of sugars, Dglucose and D-fructose, within 20-30 s and enhance food ingestion after prolonged starvation. Two candidate nutrient sensors SLC5A11 and Gr43a are required for discriminating the nutritive sugars from their nonnutritive enantiomers. Taken together, we have developed a new assay with good behavioral specificity and temporal resolution to investigate food ingestion and identify a fast-acting mechanism that senses the nutritional value of sugar.

\section{Methods}

Flies

Flies were kept in vials containing standard fly medium made of yeast, corn, and agar at $25{ }^{\circ} \mathrm{C}$ and $60 \%$ humidity and on a 12-h light:12-h dark. Canton-S virgin female flies aged for 4-6 days were used for all behavioral experiments unless otherwise indicated. SLC5A11 ${ }^{1}\left(y^{1} w^{67 c 23} ; P\{E P\right.$ gy2\}SLC5A11 ${ }^{\text {EY21708 }}$ ) mutant (hypomorphic allele) was from Bloomington (\#22498) [13]. Gr43a $a^{G A L 4}$ mutant (null allele) was from Hubert Amrein (Texas A\&M Health Science Center) [12]. HGN1-GAL4 was from Zhiqiang Yan [22]. UAS-Shibire ${ }^{t s 1}$ was from Gerald M. Rubin [17]. UAS-TRPA1 was from Paul A. Garrity [16].

\section{Chemicals}

Agar (A1296) and sucrose (S7903) were purchased from Sigma-Aldrich. D-(-)-Fructose (F0060), L(+)-Fructose (F0317), D-(+)-Glucose (G0048), and L(-)-Glucose (G0226) were purchased from TCI (Tokyo Chemical Industry). 


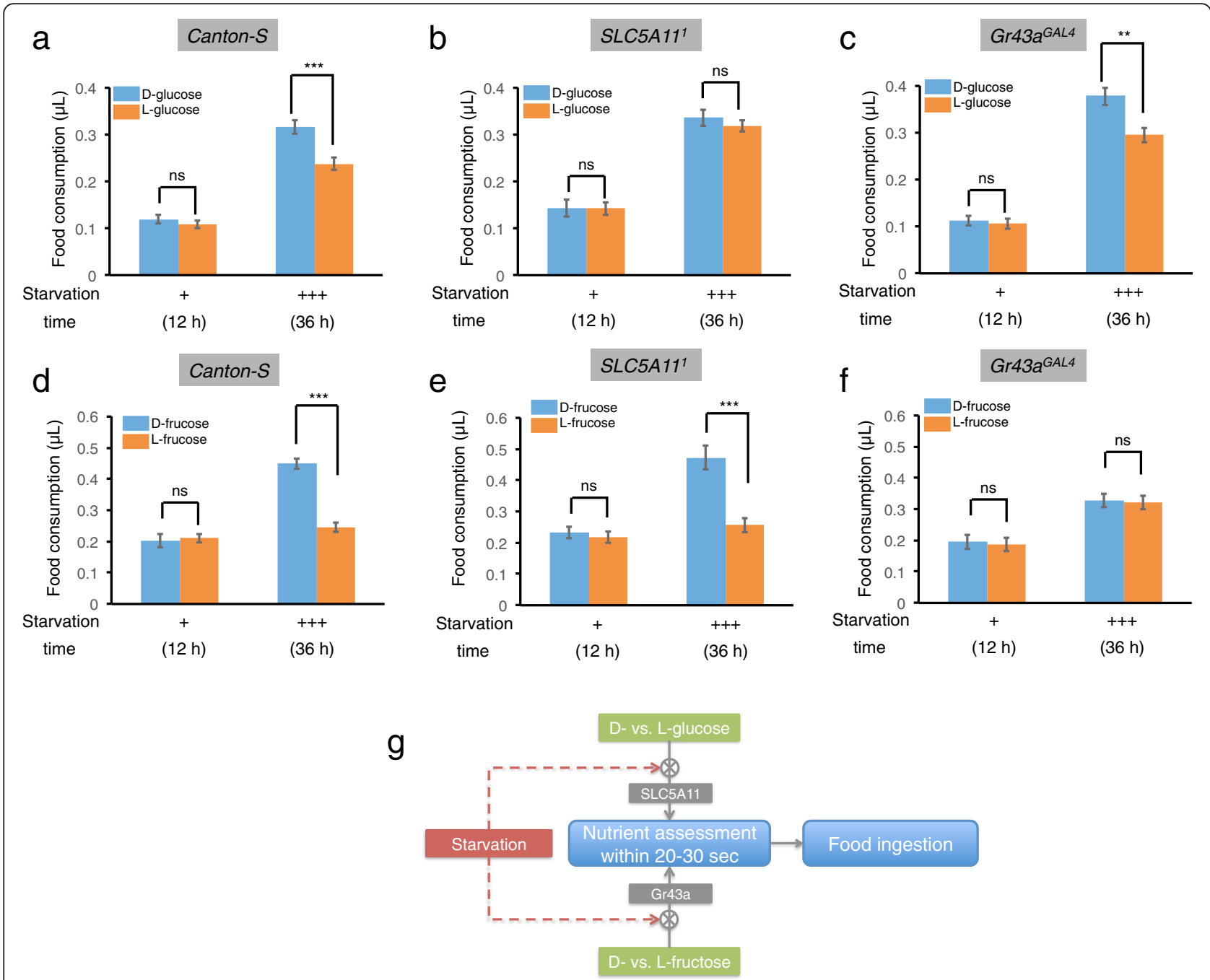

Fig. 4 Differential sensing mechanism of nutritive sugars. a-c Volume of $100 \mathrm{mM} \mathrm{D-/L-glucose} \mathrm{consumed} \mathrm{in} \mathrm{a} \mathrm{meal,} \mathrm{by} \mathrm{flies} \mathrm{with} \mathrm{indicated}$ genotypes starved for 12 and $36 \mathrm{~h}(n=24-46)$. $\mathbf{d}$-f Volume of $100 \mathrm{mM} \mathrm{D}$-/L-fructose consumed in a meal, by flies with indicated genotypes starved for 12 and $36 \mathrm{~h}(n=19-36)$. g A working model. Starved flies can assess the nutritional value of food (i.e. the presence of D- vs. Lglucose and D- vs. L-fructose) within 20-30 s and modulate food ingestion accordingly. SLC5A11 and Gr43a are required for the food nutrient assessment, respectively. Sample size for each data set was summarized in Tab. S1. Error bars represent SEM. ns, $P>0.05$; ${ }^{*} P<0.05$; ${ }^{* *} P<0.01$; ${ }^{* * *} P<0.001 ;{ }^{* * *} P<0.0001$. Student's $t$-test was used for pair wise comparisons. One-way ANOVA followed by Bonferroni post hoc test was used for comparisons for more than 2 groups. Two-way ANOVA (and post hoc test if applicable) was applied for comparisons with more than one variant

\section{MAFE assay}

Flies were fed as described previously [4]. Briefly (as illustrated in Fig. 1a-c), 4-6 days old virgin female flies were fed or wet starved in vials containing $5 \%$ sucrose plus $2 \%$ agar, or $2 \%$ agar only, before the assay, respectively. Individual flies were then gently aspirated into a $200 \mu \mathrm{L}$ pipette tip and satiated with water stimulation delivered by a pipette tip. Subsequently, $3 \mu \mathrm{L}$ liquid food (added with $5 \%$ blue dye from McCormick) in a fine graduated capillary (VWR, \#53432-604) was delivered to the proboscis of flies. As illustrated in Fig. 1a-c, if a feeding event occurred, the flies would fully extend proboscis and start drinking the liquid food. The tip of the capillary could be retrieved a bit away from the flies to allow the full extension of proboscis. Once the flies stopped feeding and retrieved proboscis, the food stimulation was repeated until the flies became unresponsive to a series of 10 food stimuli. Flies that exhibited prolonged water consumption or no PER to liquid food were excluded from the calculation of average meal size.

Before the MAFE assay, various amounts of liquid $(1 \mu \mathrm{L}, 2 \mu \mathrm{L}$, and $3 \mu \mathrm{L})$ were injected into each capillary for fine calibration (i.e. the linear correlation between the length of the liquid column in the capillary and its volume). Before and after the MAFE assay for each fly, the length of the liquid column was carefully measured 
by an electronic vernier caliper and was used to calculate the ingested volume by a single fly. The accuracy of the assay could reach $0.01 \mu \mathrm{L}$ scale.

To quantify the duration of food ingestion, two experimenters were required, with one conducting the MAFE assay while the other recording the start and stop point for each feeding bout by using a stop watch or timer.

PER. PER was assayed as described previously [4, 23]. Briefly, flies were prepared and water satiated as in the MAFE assay and subjected to different sugar solutions. Each sugar solution was presented twice, and flies showing PER to at least one of the two trials were considered positive to that concentration.

\section{Additional file}

Additional file 1: Supplementary methods. Figure S1. Starvation and food nutrient content promote feeding behavior from long-term FLIC assays. Figure S2. Starved flies also exhibited preference towards nutritive D-glucose at high concentrations. Figure S3. SLC5A11 is required for associative learning between a nutritious sugar and an odorant in a short time window. Table S1. Sample size for each data set. (PDF $1197 \mathrm{~kb}$ )

\section{Competing interests}

The authors declare that they have no competing interests.

\section{Authors' contributions}

WQ developed and characterized the MAFE assay with the help of ZY. ZY tested the mutants in the MAFE assays. ZL performed the FLIC assays. JYP and GSBS carried out the appetitive olfactory conditioning assays. LW conceived the study, participated in its design, and wrote the manuscript with help from WQ, ZY, and GSBS. All authors read and approved the final manuscript.

\section{Acknowledgements}

We thank members of the Wang lab for helpful discussions and technical assistance. We thank Hubert Amrein, Yi Rao, Zuoren Wang, Zhiqiang Yan, and Bloomington Drosophila stock center at Indiana University for fly stocks. William Ja read the manuscript before submission and offered insightful advice. Danping Chen provides scientific and administrative support in our laboratory that is crucial for our research. This study was funded in part by $\mathrm{NIH} \mathrm{RO1} \mathrm{grants}$ (NIDCD RO1DC01279, NIDDK RO1DK106636) to GSBS and the Thousand Young Talents Plan of China and the National Natural Science Foundation of China (No. 31522026) to LW.

\section{Author details}

'Life Sciences Institute, Zhejiang University, Hangzhou, Zhejiang 310058, China. ${ }^{2}$ Innovation Center for Cell Signaling Network, Zhejiang University, Hangzhou, Zhejiang 310058, China. ${ }^{3}$ Department of Cell Biology, Skirball Institute of Biomolecular Medicine, Neuroscience Institute, New York University School of Medicine, New York, NY, USA.

Received: 5 October 2015 Accepted: 14 December 2015

Published online: 21 December 2015

\section{References}

1. Pool A-H, Scott K. Feeding regulation in Drosophila. Curr Opin Neurobiol. 2014;29:57-63.

2. Gao Q, Horvath TL. Neurobiology of Feeding and Energy Expenditure. Ann Rev Neurosci. 2007;30(1):367-98.

3. Marx V. Metabolism: feeding fruit flies. Nat Meth. 2015;12(7):609-12.

4. Yang Z, Yu Y, Zhang Y, Tian Y, Qi W, Wang L. Octopamine mediates starvation-induced hyperactivity in adult Drosophila. Proc Natl Acad Sci. 2015;112(16):5219-24.
5. Root Cory M, Ko Kang I, Jafari A, Wang Jing W. Presynaptic Facilitation by Neuropeptide Signaling Mediates Odor-Driven Food Search. Cell. 2011; 145(1):133-44.

6. Ro J, Harvanek ZM, Pletcher SD. FLIC: High-Throughput, Continuous Analysis of Feeding Behaviors in Drosophila. PLoS ONE. 2014;9(6), e101107.

7. Itskov PM, Moreira J-M, Vinnik E, Lopes G, Safarik S, Dickinson MH et al. Automated monitoring and quantitative analysis of feeding behaviour in Drosophila. Nat Commun. 2014;5:4650.

8. Edgecomb RS, Harth CE, Schneiderman AM. Regulation of feeding behavior in adult Drosophila melanogaster varies with feeding regime and nutritional state. J Exp Biol. 1994;197(1):215-35.

9. Carvalho GB, Kapahi P, Benzer S. Compensatory ingestion upon dietary restriction in Drosophila melanogaster. Nat Meth. 2005;2(11):813-5.

10. Ja WW, Carvalho GB, Mak EM, de la Rosa NN, Fang AY, Liong JC et al. Prandiology of Drosophila and the CAFE assay. Proc Natl Acad Sci U S A. 2007;104(20):8253-6.

11. Dus M, Min S, Keene AC, Lee GY, Suh GSB. Taste-independent detection of the caloric content of sugar in Drosophila. Proc Natl Acad Sci. 2011;108(28):11644-9.

12. Miyamoto T, Slone J, Song $X, A$ Amrein H. A Fructose Receptor Functions as a Nutrient Sensor in the Drosophila Brain. Cell. 2012;151(5):1113-25.

13. Dus M, Ai M, Suh GSB. Taste-independent nutrient selection is mediated by a brain-specific Nat/solute co-transporter in Drosophila. Nat Neurosci. 2013;16(5):526-8.

14. Dethier V. The hungry fly. Cambridge: Harvard University Press; 1976.

15. Olds $W H, X u T$. Regulation of food intake by mechanosensory ion channels in enteric neurons. eLife. 2014;3, e04402.

16. Hamada FN, Rosenzweig M, Kang K, Pulver SR, Ghezzi A, Jegla TJ et al. An internal thermal sensor controlling temperature preference in Drosophila. Nature. 2008:454(7201):217-20.

17. Pfeiffer BD, Truman JW, Rubin GM. Using translational enhancers to increase transgene expression in Drosophila. Proc Natl Acad Sci U S A. 2012;109(17):6626-31.

18. Stafford JW, Lynd KM, Jung AY, Gordon MD. Integration of Taste and Calorie Sensing in Drosophila. J Neurosci. 2012;32(42):14767-74.

19. Reed LK, Williams S, Springston M, Brown J, Freeman K, DesRoches CE et al. Genotype-by-Diet Interactions Drive Metabolic Phenotype Variation in Drosophila melanogaster. Genetics. 2010;185(3):1009-19.

20. Fujita M, Tanimura T. Drosophila Evaluates and Learns the Nutritional Value of Sugars. Curr Biol. 2011;21(9):751-5.

21. Dus M et al. Nutrient Sensor in the Brain Directs the Action of the Brain-Gut Axis in Drosophila. Neuron. 2015;87(1):139-51.

22. Zhang $W$, Yan $Z$, Li B, Jan LY, Jan YN. Identification of motor neurons and a mechanosensitive sensory neuron in the defecation circuitry of Drosophila larvae. eLife. 2014;3, e03293.

23. Shiraiwa T, Carlson JR. Proboscis Extension Response (PER) Assay in Drosophila. J Vis Exp. 2007:3:193.

Submit your next manuscript to BioMed Central and we will help you at every step:

- We accept pre-submission inquiries

- Our selector tool helps you to find the most relevant journal

- We provide round the clock customer support

- Convenient online submission

- Thorough peer review

- Inclusion in PubMed and all major indexing services

- Maximum visibility for your research

Submit your manuscript at www.biomedcentral.com/submit 